\title{
Florida Sleeve is a Safe and Effective Technique for Valve Salvage in Acute Stanford Type A Aortic Dissection
}

\author{
Mahmoud Alhussaini ${ }^{1}$, Eric Jeng ${ }^{1}$, Tomas Martin ${ }^{1}$, Amber Filion ${ }^{1}$, Thomas Beaver ${ }^{1}$, and \\ George Arnaoutakis ${ }^{2}$ \\ ${ }^{1}$ University of Florida \\ ${ }^{2}$ Affiliation not available
}

September 25, 2021

\begin{abstract}
Objective: Valve-sparing root replacement is commonly used for management of aortic root aneurysms in elective setting, but its technical complexity hinders its broader adoption for acute Type-A Aortic Dissection (ATAAD). The Florida Sleeve (FS) procedure is a simplified form of valve sparing aortic root reconstruction that does not require coronary reimplantation. Here, we present our outcomes of the Florida Sleeve (FS) repair in patients with dilated roots in the setting of an ATAAD. Methods: We retrospectively reviewed 24 consecutive patients (2002-2018) treated with FS procedure for ATAAD. Demographic, operative, and postoperative outcomes were queried from our institutional database. Long term follow-up was obtained from clinic visits for local patients, and with telephone and telehealth measures otherwise. Results: Mean age was $49 \pm 14$ years with 19 (79\%) males. Marfan syndrome was present in 4 (16.7\%) patients and $14(58.3)$ had [?]2+ aortic insufficiency (AI). Nine (37.2\%) had preoperative mal-perfusion or shock. The FS was combined with hemi-arch replacement in $15(62.5 \%)$ patients and a zone-2 arch replacement in $9(37.5 \%)$ patients. There were $2(8.3 \%)$ early postoperative mortalities. Median follow-up period was 46 months (range; 0.3-146). The median survival of the entire cohort was 143.4 months. One patient (4.2\%) required redo aortic valve replacement for unrelated aortic valve endocarditis at 30 months postoperatively. Conclusion: FS is simplified and reproducible valve-sparing root repair. In appropriate patients, it can be applied safely in acute Stanford type-A aortic dissection with excellent early and long-term results.
\end{abstract}

Florida Sleeve is a Safe and Effective Technique for Valve Salvage in Acute Stanford Type A Aortic Dissection

Mahmoud Alhussaini MD, PhD $D^{1}$, Eric I. Jeng $M D^{2}$, Tomas D. Martin MD ${ }^{2}$,Amber Fillion ${ }^{2}$ Thomas $M$. Beaver $M D, M P H^{2}$ andGeorge J. Arnaoutakis $M D^{2}$

Cardiothoracic Surgery Department, Assiut University, Assiut, Egypt.

Division of Cardiovascular Surgery, Department of Surgery, University of Florida, Gainesville, USA.

This study was accepted at the AATS Aortic Symposium, April 23-24, 2020., New York, NY, USA.

Abstract word count : 242

Manuscript body word count : 1993

IRB approval number: \#2018-01950

Conflict of interest: none

Source of Funding: Herron Endowed Chair in Cardiothoracic Surgery at The University of Florida. 


\title{
Corresponding author :
}

George J. Arnaoutakis MD

Associate Professor

Division of Cardiovascular Surgery

University of Florida PO Box 100129

Gainesville, FL 32610

george.arnaoutakis@surgery.ufl.edu

Phone : 352-273-5501, Fax : 352-273-5593

\begin{abstract}
Objective: Valve-sparing root replacement is commonly used for management of aortic root aneurysms in elective setting, but its technical complexity hinders its broader adoption for acute Type-A Aortic Dissection (ATAAD). The Florida Sleeve (FS) procedure is a simplified form of valve sparing aortic root reconstruction that does not require coronary reimplantation. Here, we present our outcomes of the Florida Sleeve (FS) repair in patients with dilated roots in the setting of an ATAAD.
\end{abstract}

Methods: We retrospectively reviewed 24 consecutive patients (2002-2018) treated with FS procedure for ATAAD. Demographic, operative, and postoperative outcomes were queried from our institutional database. Long term follow-up was obtained from clinic visits for local patients, and with telephone and telehealth measures otherwise.

Results: Mean age was $49 \pm 14$ years with 19 (79\%) males. Marfan syndrome was present in $4(16.7 \%)$ patients and $14(58.3)$ had [?]2+ aortic insufficiency (AI). Nine (37.2\%) had preoperative mal-perfusion or shock. The FS was combined with hemi-arch replacement in $15(62.5 \%)$ patients and a zone-2 arch replacement in $9(37.5 \%)$ patients. There were $2(8.3 \%)$ early postoperative mortalities. Median follow-up period was 46 months (range; 0.3-146). The median survival of the entire cohort was 143.4 months. One patient $(4.2 \%)$ required redo aortic valve replacement for unrelated aortic valve endocarditis at 30 months postoperatively.

Conclusion: FS is simplified and reproducible valve-sparing root repair. In appropriate patients, it can be applied safely in acute Stanford type-A aortic dissection with excellent early and long-term results.

\section{Abbreviations and Acronyms:}

Ai (aortic insufficiency), AKI (Acute kidney injury), ATAAD (acute type A aortic dissection), AVR (aortic valve replacement), COPD (chronic obstructive pulmonary disease), CPB (cardiopulmonary bypass), DHCA (deep hypothermic circulatory arrest), EF (ejection fraction), FS (Florida Sleeve), LVEDD (left ventricular end diastolic diameter), LVESD (left ventricular end systolic diameter), TEE (transesophageal echocardiographic) VSRR (Valve sparing root replacement).

\section{Background}

Valve sparing root replacements (VSRR) since their introduction by Yacoub (remodeling procedure) (1) and David (reimplantation procedure) (2), have become established alternatives, in experienced hands, to composite graft replacements (Bentall procedure) for aortic root aneurysms (3). Valve sparing procedures avoid the risks of systemic thromboembolism and lifelong anticoagulation associated with mechanical valves and the risks of structural valve deterioration associated with bioprosthetic valves and have excellent clinical outcomes and good long-term durability $(1,4)$.

Acute type-A aortic dissection (ATAAD) is a life-threatening emergency and the primary goal of surgery is to save the life of the patient. For dissections involving the aortic root, the Bentall procedure has traditionally been considered the gold standard owing to its technical feasibility and reproducibility (5). There are several 
reports of VSRR techniques in ATAAD with acceptable early and long term results (3, 6-8). However, the surgical complexity of VSRR and the longer cross-clamp time has slowed wider adoption in the emergent setting (9).

The Florida Sleeve (FS) procedure is a simplified valve sparing root repair that does not require coronary artery reimplantation (10). The long term durability of this type of repair was published recently yielding excellent results (11). Moreover, the biomechanical characteristics of the FS leads to lower aortic valve stress and prevents possible aortic root distortion or harmful aortic wall stresses (12). Our aim is to present our outcomes with the FS in ATAAD.

\section{Patients and methods}

\section{Patients}

The University of Florida Institutional Review Board approved this study (\#2018-01950). A waiver of informed consent was granted. We retrospectively reviewed 24 consecutive patients at UF Health Aortic Disease Center (The University of Florida College of Medicine, Gainesville, FL) treated with FS procedure for ATAAD from (2002-2018). FS was applied by surgeon discretion and indicated when there was an associated root dilatation (aortic root diameter $>4.5 \mathrm{~cm}$ and/or $>4 \mathrm{~cm}$ in patients $<60$ years or with connective tissue disorder) in the setting of ATAAD.

\section{Operative technique}

The details of the FS procedure have been described previously $(10,11,13)$. Briefly, cardiopulmonary bypass (CPB) was commenced using either axillary, femoral, or central cannulation using the Seldinger technique and under transesophageal echocardiographic (TEE) guidance. After cooling to a core body temperature of $18^{\circ} \mathrm{C}$ and flat line EEG, deep hypothermic circulatory arrest (DHCA) was initiated with or without retrograde cerebral perfusion. he extent of distal aortic repair was determined. Hemiarch replacement was utilized for intimal tears in the ascending aorta. Zone 2 arch replacements were performed for tears in the arch and proximal descending aorta and/or in younger patients where there was concern for distal aortic degeneration and high potential for secondary intervention. After de-airing of the distal aortic graft, re-establishing of $\mathrm{CPB}$ and during the rewarming phase, the aortic root was addressed. The aortic root was dissected circumferentially to the annular level, and the proximal portions of the coronary arteries were mobilized. To size graft appropriately, a Hegar dilator is used to measure the annulus directly, and a Valsalva graft $6-8 \mathrm{~mm}$ larger than the annulus diameter is selected, typically 30 or $32 \mathrm{~mm}$ graft for women and 32 or $34 \mathrm{~mm}$ graft for men. If additional annular reduction is desired in patients with larger annulus size, an additional fourth annuloplasty suture is placed at nadir of noncoronary cusp. Typically, three subannular 2-0 pledgeted Ethibond (Ethicon US, LLC, Cincinnati) mattress sutures are utilized to anchor the FS graft at the root (three in the subcommissural positions, and additional fourth subannular suture at the nadir of the noncoronary cusp) (Figure-1A ). With bicuspid valves, subannular sutures were placed under each of the commissures. We were careful with additional subannular sutures in bicuspid valves as they may produce unwanted leaflet elongation, compromising the geometry of coaptation and subsequently valvular competence. Locations of the coronary arteries were marked on the graft, and vertical slits were made to create coronary keyholes. The subannular anchor sutures were placed through a Valsalva graft (Terumo Aortic Vascutek Ltd., Renfrewshire, Scotland, UK), graft was seated, and the sutures are tied with a Hegar dilator (Jarit Instruments, Hawthorne, NY) through the annulus to prevent a purse-string effect. The slits below the coronary arteries were closed using simple sutures making sure these keyholes were large enough to accommodate the coronary arteries. At this time, the Valsalva graft is cut to size and supra-commissural sutures were placed to resuspend the valve, being careful to reapproximate all of the dissected layers of the aorta (Figure-1B ). The dissection repair is completed with sandwich of Teflon-felt - aorta - Dacron at the sinotubular junction (STJ) with a running horizontal mattress (Figure-1C ). A graft to graft anastomosis of the proximal and distal repairs completes the operation (Figure-1D ). Intraoperative TEE was used to assess the quality of valve repair, degree of aortic insufficiency (AI) and ventricular function to detect any segmental wall motion abnormalities that might have happened due to coronary artery impingement. 


\section{Endpoints and Statistical analysis}

The primary endpoints chosen were the early mortality and postoperative AI. Additional endpoints included procedural safety, long-term durability, and freedom from reoperation. Postoperative mortality was defined as mortality at 30 days postoperatively or at time of discharge. Acute kidney injury (AKI) was defined using the AKIN Criteria (14).We used echocardiography to assess the degree of AI (graded as: $0=$ none, $1+=$ trace/minimal, $2+=$ mild, $3+=$ moderate, and $4+=$ severe), ejection fraction (EF), left ventricular end diastolic diameter (LVEDD) and left ventricular end systolic diameter (LVESD). Follow-up echocardiography measurements were not available for all patients because some patients had postoperative follow-up echocardiography at outside centers. Long-term follow-up was obtained from clinic visits for local patients, and with telephone and telehealth measures otherwise. Social security death index was utilized for those who could not be contacted.

Continuous variables were presented as the mean +- standard deviation (SD) and categorical data as frequency and percentage. Quantitative measures are expressed as mean +- one standard deviation where appropriate. Outcome data were analyzed with Chi squared test, Fisher exact test, and the independent Student $t$ test where appropriate. Differences were considered significant if a $95 \%$ confidence interval was achieved. Patients' survival rate and freedom from reoperation were evaluated by Kaplan-Meier and lifetable methods. All statistics were performed by Graphpad Prism software (Version 8.4, Graphpad software, LLC, San Diego).

\section{Results}

Patients demographics and preoperative variables

Twenty-four patients treated with FS procedure for ATAAD, 19 men (79.2\%) with mean age of $49+-14$ years were included in the study. Marfan syndrome was present in $4(16.7 \%)$ patients and $12(50 \%)$ had $>2+$ aortic insufficiency (AI). Nine (37.2\%) had preoperative malperfusion or shock. Mean aortic root diameter was $53.3+-9 \mathrm{~mm}$. The main preoperative features of the patients are summarized in Table 1.

Operative variables and postoperative outcomes

Operative and postoperative variables are demonstrated in Table 2. The FS was combined with hemiarch replacement in 15 (62.5\%) patients or arch debranching and zone-2 arch replacement in $9(37.5 \%)$ patients. Mean CPB time was 225.5 +- 45.8 minutes and mean myocardial ischemic time was 137.4 +35.5 minutes. DHCA was used in all patients and this was combined with either antegrade or retrograde cerebral perfusion in $15(62.5 \%)$ patients. Intraoperative TEE showed procedural success in all patients regarding AI and none of the patients had more than mild AI at time of discharge. None of the patients had postoperative neurological complications or required re-exploration for bleeding. There were $2(8.3 \%)$ early postoperative mortalities; one had preoperative renal failure and myocardial infarction and the other had a preoperative ruptured ascending aorta and cardiac tamponade with malperfusion and the postoperative course was complicated by septicemia, renal and respiratory failure.

\section{Follow-up}

The median follow-up period was 46 months (range; 0.3-146). Only $1(4.2 \%)$ required redo aortic valve replacement (AVR) for endocarditis (30 months postoperatively) during follow-up (Figure-2). Five (20.8\%) patients required intervention on the descending thoracic/thoracoabdominal aorta during follow-up. There were 2 late postoperative mortalities one from respiratory failure complicating COPD 12 years after surgery and the other from sepsis complicating open repair of ruptured thoracoabdominal aneurysm 3 years following ATAAD repair. Echocardiography during follow-up was available in 12 patients at 1-3years and it showed decrease of mean degree of AI from 2.4+-1.6 preoperatively to 0.9+-1.4 at 1-3years $(P=0.01)$ and LVEDD from $54.7+-6.9 \mathrm{~mm}$ preoperatively to $46.9+-9.4 \mathrm{~mm}$ at 1 -3years $(P=0.04)$ (table-3). Freedom from redo 1,5 and 10 years AVR is estimated to be $100 \%, 93+-6.9 \%$ and $93+-6.9 \%$ respectively. Estimated median survival time is 143.4 months and estimated survival is $85.2+-8 \%$ at 1 and 10 years (Figure-3). 


\section{Discussion}

Although VSRRs are well-established surgical options for management of elective aortic root pathology (chronic dissections or aneurysms), it is not widely performed in the setting of ATAAD with root pathology. VSRR procedures are hindered by their technical complexity and the additional time required to perform the repair and may be not suitable in this emergent clinical setting. Furthermore, there are concerns about the safety and durability and the risk of late valve dysfunction secondary to Aortic Insufficiency.

The immediate goal in ATAAD is to save the life of the patient and in select cases root replacement with composite valve conduit is considered traditional therapy. Even at centers with extensive VSRR experience VSSR in dissection is limited as in Toronto with a quarter of a century of experience there were 28 patients with ATAAD (4). The largest experience with David procedure in ATAAD was reported by the Hannover group. They reported 109 David I procedures in ATAAD with $11 \%$ operative mortality and $85 \%$ freedom from valve-related reoperation at 10 years (15). The Leipzig group reported 78 VSRRs (51 Yacoub and 27 David) with 30-day mortality of $15 \%, 8$-year survival of $55 \%$ and estimated freedom from reoperation 8.9 years (16). Interestingly, only 2 of their patients (2.6\%) had Marfan syndrome (David procedures) and this may explain their preferred use of the Yacoub technique. Recently, Aubin and his colleagues reported their results with 28 David repairs in TAAD with 30-day and 5-year mortality of $18 \%$ and $29 \%$, respectively (17).

The Florida Sleeve is a simplified aortic root repair that does not involve replacement of the sinuses of Valsalva. Instead, the entire root unit is enveloped in a Valsalva Dacron graft tailored as a "sleeve", leaving the coronary artery ostia intact. This allows reduction and stabilization of the virtual basal ring and the STJ, while the Valsalva graft allows for normal expansion of the sinuses of Valsalva during cardiac cycle, rendering more physiological biomechanics than the David procedure - with lower leaflet and commissural stress as reported by Gamba and his colleagues (12). Furthermore, this technique does not require coronary button reimplantation, which can be treacherous if the friable dissection plane extends into either, or both, of the coronary ostia. Our group recently published long-term experience with the FS technique with $98 \%$ freedom from reoperation and $93 \%$ survival at 8 years (11). This is comparable to the long-term experience of David and his colleagues with VSRRs who reported $97 \%$ freedom from reoperation and $89 \%$ survival at 10 years (4).

The $8 \%$ mortality with FS repair in ATAAD in this series is favorable compared to a recent International Registry of Acute Aortic Dissection (IRAD) report with 18\% 30-day mortality in ATAAD (9). Procedural success in the form of absence of greater than $2+$ degree AI was achieved in all patients. Only one patient required AVR during follow-up but that was due to valve endocarditis suggesting the FS is a stable aortic valve repair for the long term. Echocardiographic data showed improvement in LVEDD at 1-3 years which is thought to be the most important indicator of AI (18). Freedom of redo AVR (92.9\%) at 10 years and 10 year survival $(85.2 \%)$ is comparable to other VSRRs in type A dissection (15-17).

\section{Limitations}

This study has the following limitations: 1) It is a retrospective single center study of a small group of patients. 2) Follow-up echocardiography was available in only $50 \%$ of the study group.

\section{Conclusion:}

The Florida Sleeve is a simplified and reproducible valve-sparing root repair. In carefully selected patients, it can be applied safely in ATAAD with excellent early and long-term results. Further studies are needed comparing larger numbers of patients to composite valved conduit root replacements and other valve sparing root repairs.

1. Yacoub MH, Gehle P, Chandrasekaran V, Birks EJ, Child A, Radley-Smith R. Late results of a valvepreserving operation in patients with aneurysms of the ascending aorta and root. J Thorac Cardiovasc Surg. 1998;115(5):1080-90.

2. David TE, Feindel CM. An aortic valve-sparing operation for patients with aortic incompetence and 
aneurysm of the ascending aorta. J Thorac Cardiovasc Surg. 1992;103(4):617-21; discussion 22.

3. Ouzounian M, Rao V, Manlhiot C, Abraham N, David C, Feindel CM, et al. Valve-Sparing Root Replacement Compared With Composite Valve Graft Procedures in Patients With Aortic Root Dilation. J Am Coll Cardiol. 2016;68(17):1838-47.

4. David TE, Feindel CM, David CM, Manlhiot C. A quarter of a century of experience with aortic valvesparing operations. J Thorac Cardiovasc Surg. 2014;148(3):872-9; discussion 9-80.

5. Hagan PG, Nienaber CA, Isselbacher EM, Bruckman D, Karavite DJ, Russman PL, et al. The International Registry of Acute Aortic Dissection (IRAD): new insights into an old disease. JAMA. 2000;283(7):897903.

6. Lee H, Cho YH, Sung K, Kim WS, Park KH, Park PW, et al. Clinical outcomes of valve-sparing root replacement in acute type A aortic dissection. Scand Cardiovasc J. 2015;49(6):331-6.

7. Stephens EH, Borger MA. Getting to the root of the matter: management of the aortic root in Type A aortic dissection. Eur J Cardiothorac Surg. 2016;50(2):230-1.

8. Tanaka H, Ikeno Y, Abe N, Takahashi H, Inoue T, Okita Y. Outcomes of valve-sparing root replacement in acute Type A aortic dissection. Eur J Cardiothorac Surg. 2018;53(5):1021-6.

9. Evangelista A, Isselbacher EM, Bossone E, Gleason TG, Eusanio MD, Sechtem U, et al. Insights From the International Registry of Acute Aortic Dissection: A 20-Year Experience of Collaborative Clinical Research. Circulation. 2018;137(17):1846-60.

10. Hess PJ, Jr., Klodell CT, Beaver TM, Martin TD. The Florida sleeve: a new technique for aortic root remodeling with preservation of the aortic valve and sinuses. Ann Thorac Surg. 2005;80(2):748-50.

11. Aalaei-Andabili SH, Martin TD, Hess PJ, Karimi A, Bavry AA, Arnaoutakis GJ, et al. The Florida Sleeve Procedure Is Durable and Improves Aortic Valve Function. Aorta (Stamford). 2019;7(2):49-55.

12. Tasca G, Selmi M, Votta E, Redaelli P, Sturla F, Redaelli A, et al. Aortic Root Biomechanics After Sleeve and David Sparing Techniques: A Finite Element Analysis. Ann Thorac Surg. 2017;103(5):1451-9.

13. Hess PJ, Jr., Harman PK, Klodell CT, Beaver TM, Bologna MT, Mikhail P, et al. Early outcomes using the Florida sleeve repair for correction of aortic insufficiency due to root aneurysms. Ann Thorac Surg. 2009;87(4):1161-8; discussion 8-9.

14. Mehta RL, Kellum JA, Shah SV, Molitoris BA, Ronco C, Warnock DG, et al. Acute Kidney Injury Network: report of an initiative to improve outcomes in acute kidney injury. Crit Care. 2007;11(2):R31.

15. Beckmann E, Martens A, Pertz J, Kaufeld T, Umminger J, Hanke JS, et al. Valve-sparing David I procedure in acute aortic type A dissection: a 20-year experience with more than 100 patients. Eur J Cardiothorac Surg. 2017;52(2):319-24.

16. Subramanian S, Leontyev S, Borger MA, Trommer C, Misfeld M, Mohr FW. Valve-sparing root reconstruction does not compromise survival in acute type A aortic dissection. Ann Thorac Surg. 2012;94(4):12304 .

17. Aubin H, Akhyari P, Rellecke P, Pawlitza C, Petrov G, Lichtenberg A, et al. Valve-Sparing Aortic Root Replacement as First-Choice Strategy in Acute Type a Aortic Dissection. Front Surg. 2019;6:46.

18. Nishimura RA, Otto CM, Bonow RO, Carabello BA, Erwin JP, 3rd, Guyton RA, et al. 2014 AHA/ACC guideline for the management of patients with valvular heart disease: a report of the American College of Cardiology/American Heart Association Task Force on Practice Guidelines. J Thorac Cardiovasc Surg. 2014;148(1):e1-e132.

Table-1: Patients demographics and preoperative characteristics 


\begin{tabular}{ll}
\hline Variable & $\mathrm{N}=24(\%)$ \\
\hline Age (years) mean $\pm \mathrm{SD}$ & $49.25 \pm 14.04$ \\
Male & $19(79.2)$ \\
Body mass index mean $\pm \mathrm{SD}$ & $28.4 \pm 6.9$ \\
Type of dissection (Debakey classification) & \\
I & $21(87.5)$ \\
II & $3(12.5)$ \\
Marfan syndrome & $4(16.7)$ \\
Bicuspid aortic valve (BAV) & $1(4.2)$ \\
Familial aortic aneurysms (non-marfan) & $3(12.5)$ \\
Current or ex-smoker & $13(54.5)$ \\
Chronic lung disease & $3(12.5)$ \\
Diabetes & $0(0)$ \\
Hypertension & $14(58.3)$ \\
Dyslipidemia & $3(12.5)$ \\
Peripheral arterial disease & $2(8.3)$ \\
Cerebrovascular disease & $2(8.3)$ \\
Chronic renal insufficiency & $3(12.5)$ \\
Renal insufficiency on dialysis & $1(4.2)$ \\
Arrhythmia & $1(4.2)$ \\
Congestive heart failure & $1(4.2)$ \\
Previous sternotomy & $1(4.2)$ \\
Degree of aortic insufficiency $(\mathrm{AI})$ & \\
0 & $5(20.8)$ \\
1+ & $2(8.3)$ \\
2+ & $5(20.8)$ \\
3+ & $3(12.5)$ \\
Condition at time of surgery & $9(37.2)$ \\
Aortic root diameter (millimeter) mean $\pm \mathrm{SD}$ & $53.3 \pm 9$ \\
Preoperative myocardial infarction & $1(4.2)$ \\
Malperfusion syndrome & $9(37.2)$ \\
Bloody pericardial effusion & $9(37.5)$ \\
Preoperative hematocrit (\%) mean $\pm \mathrm{SD}$ & $35.7 \pm 8.7$ \\
Preoperative creatinine (mg/dl) mean $\pm \mathrm{SD}$ & $1.4 \pm 1.3$ \\
Preoperative INR mean \pm SD & $1.1 \pm 0.2$ \\
\hline & \\
&
\end{tabular}

INR; international normalized ratio

Table-2 Operative variables, postoperative outcomes and follow-up

\begin{tabular}{ll}
\hline Variable & $\mathrm{N}=24(5)$ \\
\hline Type of arch repair & $15(62.5)$ \\
Hemi-arch replacement & $9(37.5)$ \\
Arch debranching and zone 2 arch replacement & $1(4.2)$ \\
Antegrade stent graft deployment (frozen elephant & \\
trunk) & $2(8.3)$ \\
Concomitant coronary artery bypass grafting & \\
(CABG) & \\
Florida sleeve Valsalva graft size &
\end{tabular}




\begin{tabular}{|c|c|}
\hline Variable & $\mathrm{N}=24(5)$ \\
\hline $32 \mathrm{~mm}$ & $20(83.3)$ \\
\hline $34 \mathrm{~mm}$ & $3(12.5)$ \\
\hline $36 \mathrm{~mm}$ & $1(4.2)$ \\
\hline $\begin{array}{l}\text { Cardiopulmonary bypass time (minutes) mean } \pm \\
\text { SD }\end{array}$ & $225.5 \pm 45.8$ \\
\hline $\begin{array}{l}\text { Myocardial ischemic time (minutes) mean } \pm \mathrm{SD} \\
\text { Arterial cannulation type }\end{array}$ & $137.4 \pm 35.5$ \\
\hline Central cannulation & $18(75)$ \\
\hline Femoral cannulation & $6(25)$ \\
\hline Circulatory arrest & $24(100)$ \\
\hline Cerebral perfusion & $15(62.5)$ \\
\hline Antegrade cerebral perfusion (ACP) & $7(29.2)$ \\
\hline Retrograde Cerebral perfusion (RCP) & $7(29.2)$ \\
\hline Combined ACP/RCP & $1(4.2)$ \\
\hline Circulatory arrest time (minutes) mean $\pm \mathrm{SD}$ & $38.8 \pm 23.5$ \\
\hline Cerebral ischemic time (minutes) mean $\pm \mathrm{SD}$ & $18.7 \pm 12.1$ \\
\hline \multicolumn{2}{|l|}{ Postoperative outcomes } \\
\hline \multicolumn{2}{|l|}{ Postoperative degree of Aortic insufficiency (AI) } \\
\hline $0-1$ & $21(75)$ \\
\hline 2 & $3(25)$ \\
\hline $3-4$ & $0(0)$ \\
\hline Postoperative acute kidney injury & $5(20.8)$ \\
\hline $\begin{array}{l}\text { Postoperative renal failure necessitating dialysis at } \\
\text { discharge }\end{array}$ & $3(12.5)$ \\
\hline Arrhythmia & $4(16.7)$ \\
\hline Postoperative stroke & $0(0)$ \\
\hline Postoperative bleeding necessitating re-exploration & $0(0)$ \\
\hline Early postoperative mortality & $2(8.3)$ \\
\hline $\mathrm{ICU}$ stay (days) mean $\pm \mathrm{SD}$ & $8.1 \pm 8.4$ \\
\hline $\begin{array}{l}\text { Postoperative mechanical ventilation time (hours) } \\
\text { mean } \pm \mathrm{SD}\end{array}$ & $114.4 \pm 208$ \\
\hline Length of hospital stay (days) mean $\pm \mathrm{SD}$ & $12 \pm 8.2$ \\
\hline $\begin{array}{l}\text { >mild aortic insufficiency in postoperative } \\
\text { echocardiography }\end{array}$ & $0(0)$ \\
\hline \multicolumn{2}{|l|}{ Follow-up } \\
\hline Follow-up duration (months) mean $\pm \mathrm{SD}$ & $53.7 \pm 50.5$ \\
\hline Redo aortic valve replacement (AVR) & $1(4.2)$ \\
\hline TEVAR & $3(12.5)$ \\
\hline Open descending/thoracoabdominal aortic repair & $2(8.3)$ \\
\hline Estimated survival time (months) mean \pm SEM & $125.2 \pm 10.6$ \\
\hline
\end{tabular}

ICU; intensive care unit, TEVAR; thoracic endovascular aortic repair

Table-3 Preoperative, early postoperative, and last follow up Echocardiographic data.

\begin{tabular}{llll}
\hline & Preoperative echocardiography & Early postoperative echocardiography & Last follow-up echoc \\
\hline Degree of AI mean \pm SD & $2.4 \pm 1.6$ & $0.3 \pm 0.7$ & $0.9 \pm 1.4$ \\
LVEDD $(\mathrm{mm})$ mean \pm SD & $54.7 \pm 6.9$ & $47.5 \pm 7.6$ & $46.9 \pm 9.4$ \\
LVESD $(\mathrm{mm})$ mean \pm SD & $38.5 \pm 5.9$ & $31.9 \pm 7.9$ & $32.1 \pm 6.5$
\end{tabular}




\begin{tabular}{llll}
\hline & Preoperative echocardiography & Early postoperative echocardiography & Last follow-up echoc \\
\hline $\mathrm{EF}(\%)$ mean $\pm \mathrm{SD}$ & $56.3 \pm 9.6$ & $59.7 \pm 6.9$ & $63.43 \pm 4.2$ \\
\hline
\end{tabular}

AI; aortic insufficiency, LVEDD; left ventricular end diastolic diameter, LVESD; left ventricular end systolic diameter, EF; ejection fraction

\section{Figure legends}

- Figure 1A showing four subannular 2-0 pledgeted mattress sutures at the aortic root (three in the subcommisural positions, and one at the nadir of the left-non coronary cusp). These sutures are anchored to the Valsalva graft.

- Figure 1B The slits below the coronary arteries were closed using simple sutures. The Valsalva graft is cut at the level of the STJ and 3 resuspension sutures are paced at each commissure to ensure valve competence.

- Figure 1C Running horizontal mattress sutures incorporating the Dacron graft, dissected layers of the aorta and Teflon felt strip.

- Figure 1D Restoring the aortic continuity with graft to graft anastomosis.

- Figure-2 Freedom from aortic valve reoperation following Florida Sleeve repair for acute type A aortic dissection.

- Figure-3 Kaplan-Meier survival curve following Florida Sleeve repair for acute type A aortic dissection.

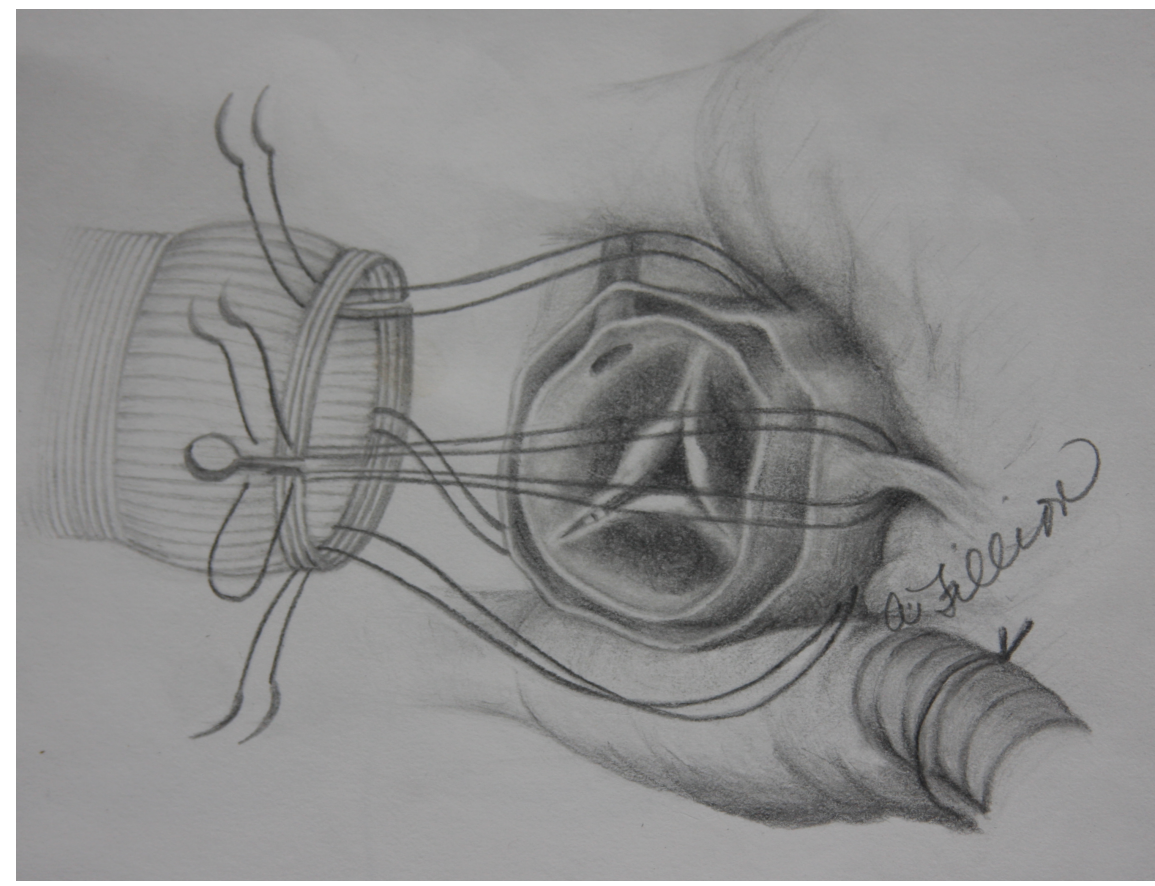




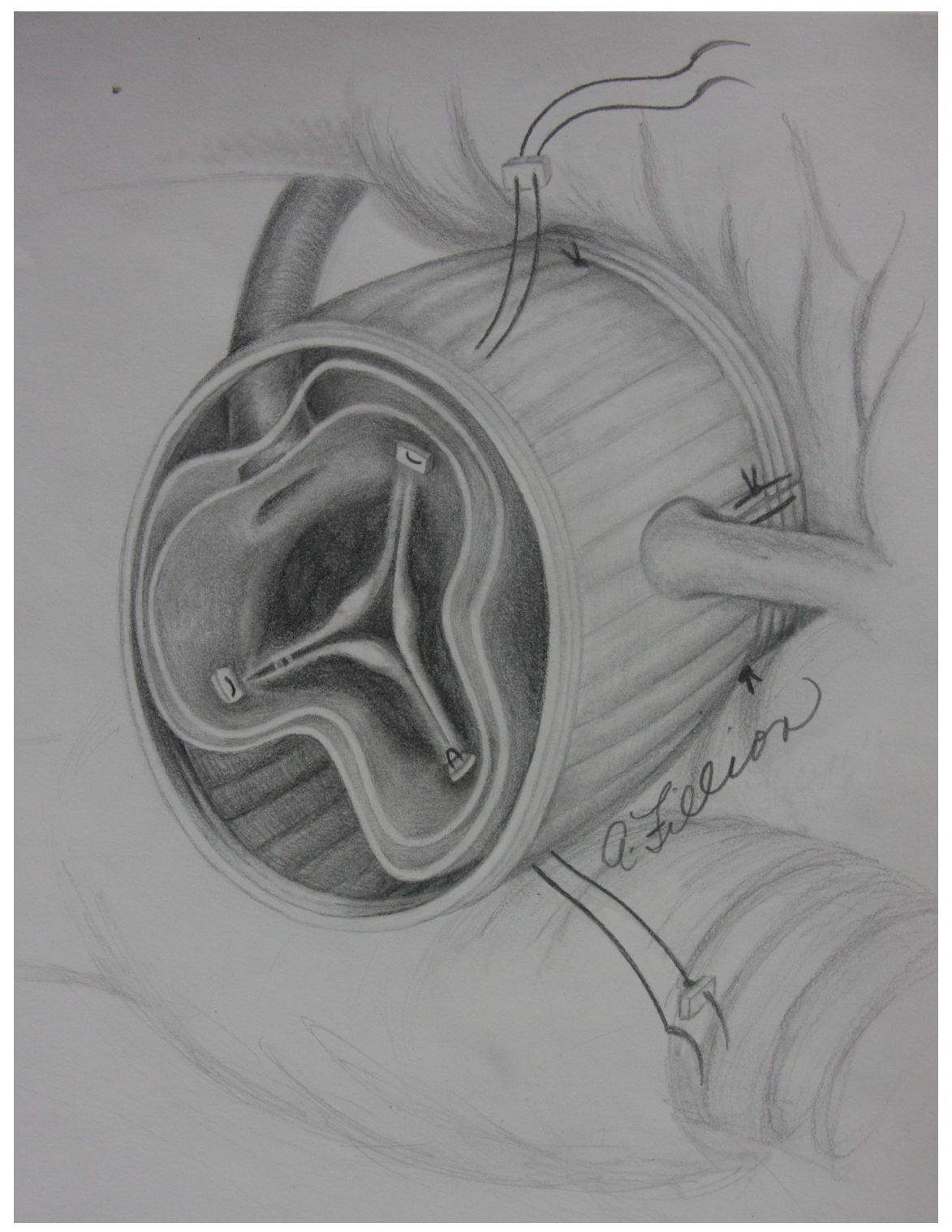




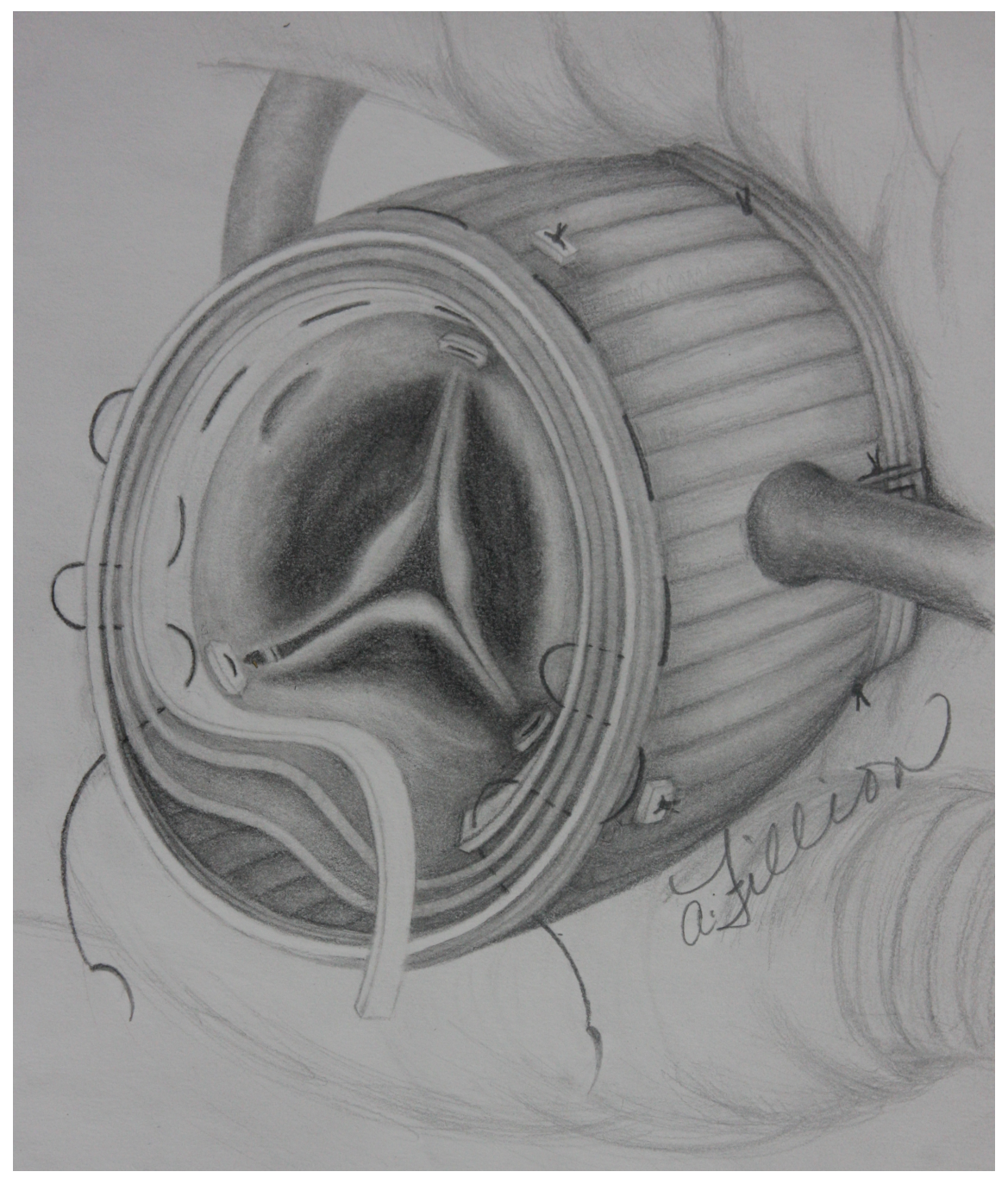



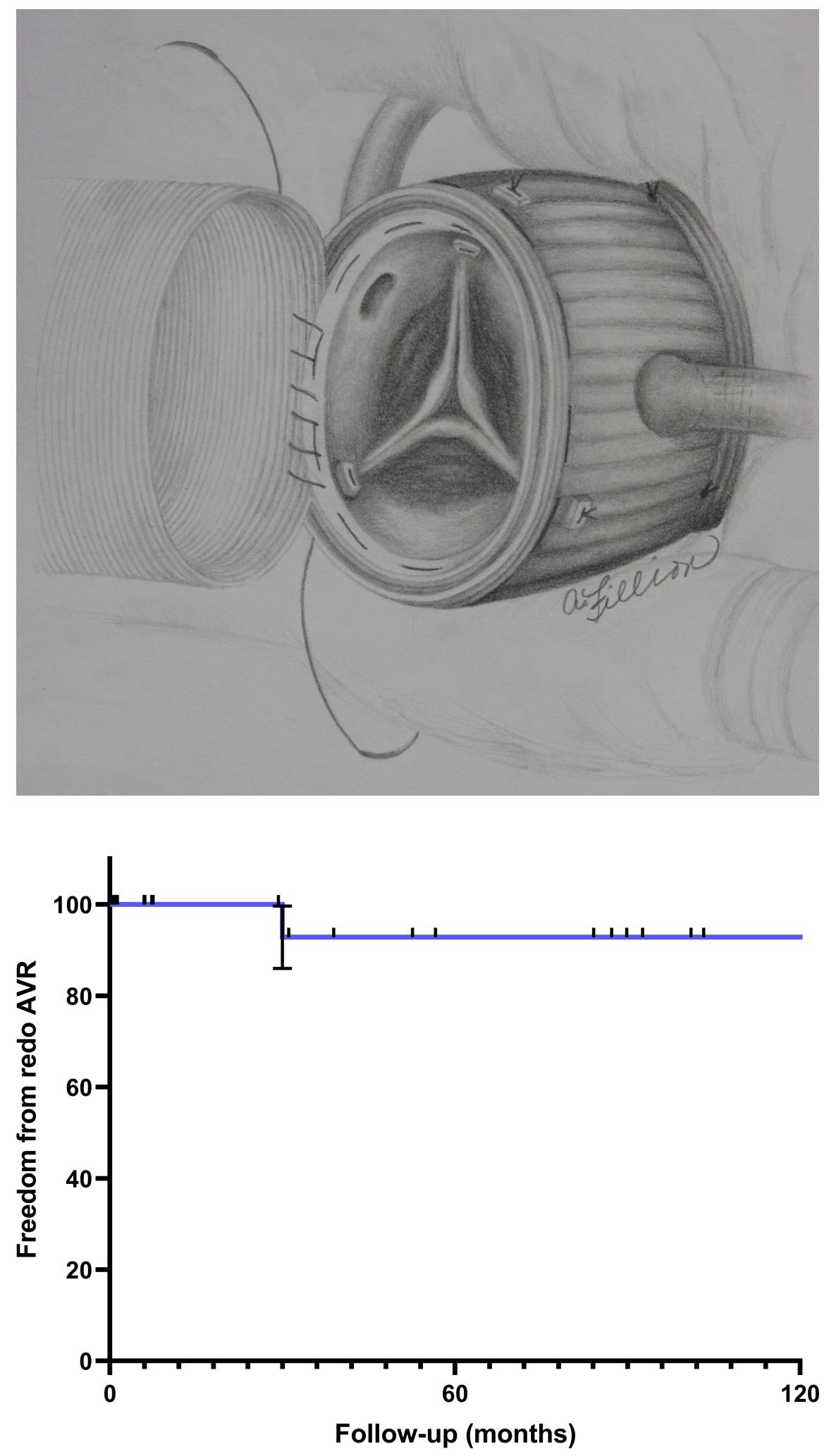


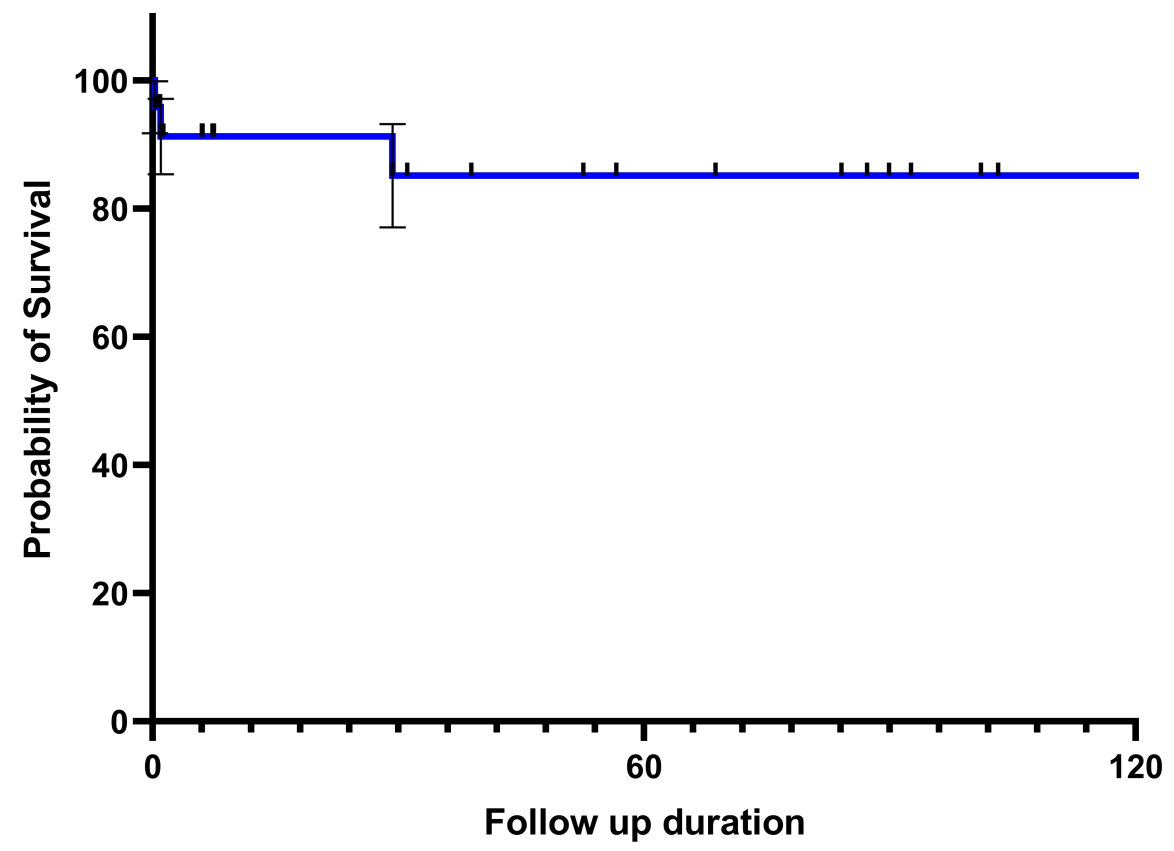

